\title{
INDOOR A* PATHFINDING THROUGH AN OCTREE REPRESENTATION OF A POINT CLOUD
}

\author{
O.B.P.M. Rodenberg ${ }^{\mathrm{a}}$, E. Verbree $^{\mathrm{b}} *$, S. Zlatanova $^{\mathrm{c}}$
}

\author{
${ }^{a}$ Delft University of Technology, Faculty of Architecture and the Built Environment, MSc Geomatics, \\ Julianalaan 134, 2628 BL, Delft, The Netherlands, orodenberg@ gmail.com \\ ${ }^{\mathrm{b}}$ Delft University of Technology, Faculty of Architecture and the Built Environment, \\ OTB - Research Institute for the Built Environment, GIS Technology, \\ Julianalaan 134, 2628 BL, Delft, The Netherlands, e.verbree@tudelft.nl \\ ${ }^{c}$ Delft University of Technology, Faculty of Architecture and the Built Environment, \\ Department of Urbanism, 3D Geoinformation, \\ Julianalaan 134, 2628 BL, Delft, The Netherlands, s.zlatanova@tudelft.nl
}

\section{Commission IV}

KEY WORDS: Indoor pathfinding, octree, network graph, connectivity, point cloud, collision avoidance

\begin{abstract}
:
There is a growing demand of 3D indoor pathfinding applications. Researched in the field of robotics during the last decades of the $20^{\text {th }}$ century, these methods focussed on $2 \mathrm{D}$ navigation. Nowadays we would like to have the ability to help people navigate inside buildings or send a drone inside a building when this is too dangerous for people. What these examples have in common is that an object with a certain geometry needs to find an optimal collision free path between a start and goal point.

This paper presents a new workflow for pathfinding through an octree representation of a point cloud. We applied the following steps: 1) the point cloud is processed so it fits best in an octree; 2) during the octree generation the interior empty nodes are filtered and further processed; 3 ) for each interior empty node the distance to the closest occupied node directly under it is computed; 4) a network graph is computed for all empty nodes; 5) the A* pathfinding algorithm is conducted.

This workflow takes into account the connectivity for each node to all possible neighbours (face, edge and vertex and all sizes). Besides, a collision avoidance system is pre-processed in two steps: first, the clearance of each empty node is computed, and then the maximal crossing value between two empty neighbouring nodes is computed. The clearance is used to select interior empty nodes of appropriate size and the maximal crossing value is used to filter the network graph. Finally, both these datasets are used in A* pathfinding.
\end{abstract}

\section{INTRODUCTION}

There is a growing demand of 3D indoor pathfinding applications (Isikdag et al., 2013). Researched in the field of robotics during the last decades of the $20^{\text {th }}$ century, these methods focussed on 2D navigation. Besides the algorithms were developed for relatively slow robots. Nowadays we would like to have the ability to help people navigate inside buildings or send a drone inside a building when this is too dangerous for people.

What these examples have in common is that an object with a certain geometry needs to find an optimal collision free path between a start and goal point. To manage this we need to know the geometry of the object and a model of the environment. One way of representing the latter one can be using a point cloud. However, a point cloud of the environment alone does not give enough information to find a route: for this, the empty (pointless) space is needed. The empty space can be derived from the occupied space by segmenting a point cloud.

A common method to structure and segment a point cloud is through an octree data structure. An octree consists out of a cubical volume which is recursively subdivided 'into eight congruent disjoint cubes (called octants) until blocks of a uniform colour are obtained, or a predetermined level of decomposition is reached' (Samet, 1982). Here, 'uniform colour' indicates: the block is completely empty or completely occupied. They are used for the partitioning of space and result in a hierarchical tree structure. This makes operations like neighbour finding and indexing efficient (Vörös, 2000).

The octree in Figure 1 is created from a point cloud of 2.196.903 points with 6 octree levels.
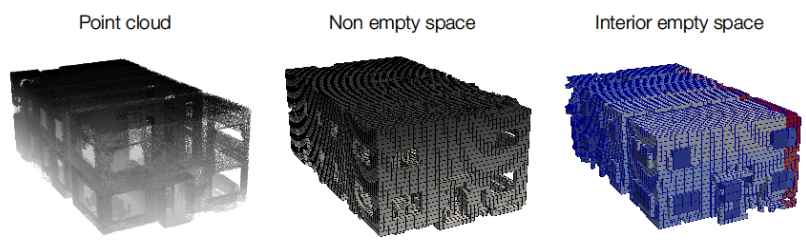

Figure 1: Process of point cloud to empty space octree

One of the advantages of an octree is efficient structuring of space, large empty space can be represented by a large node high in the octree, as shown in Figure 2 by a quadtree, the 2D counterpart of an octree.

These large empty nodes reduce the amount of nodes in the octree. This is an advantageous property of an octree for pathfinding. The large nodes reduces the amount of nodes and subsequently the possibilities to discover in pathfinding.

The goal of this research is to identify the effects of geometrical point cloud operations, octree operators and $\mathrm{A}^{*}$ operations on $\mathrm{A}^{*}$ pathfinding. 
The remainder of this paper is structured as follows: the second section provides related work; section 3 presents the methodology of the research; section 4 presents the tests and results, and finally, section 5 discusses the validation tests and concludes on the findings.
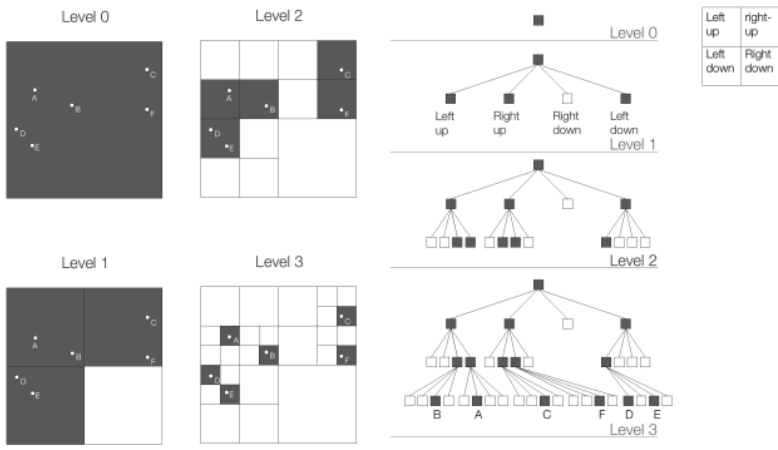

Figure 2: Subdivision of a quadtree, large empty space can be constructed out of a single quadrant.

\section{RELATED WORK}

The related work of this research is threefold: pathfinding methods in octree structures, neighbour finding methods in octree data structures and finally collision avoidance methods.

\subsection{Pathfinding methods in octree structures}

Herman (1986) present a combination of two pathfinding methods in an octree representation. It combines a hill climbing (local optimisation) method in combination with an $\mathrm{A}^{*}$ algorithm. The $\mathrm{A}^{*}$ algorithm is an extension of the Dijkstra algorithm. The $\mathrm{A}^{*}$ algorithm introduces a heuristic cost, which approximates the cost from the marked node to the goal node (Hart et al., 1968).

Hill climbing is able to quickly compute a path because it only uses the distances to adjacent nodes to decide the next node. However, this method has a tendency to stick in ' $U$ ' shaped obstacles. When this happen an $\mathrm{A}^{*}$ algorithm is used until the obstacle is avoided. This method is fast, although it trends to move away from the shortest route.

Vörös (2001) uses a hill climbing method in combination with a distance map to compute a path in a quadtree and octree representation. By using an octree representation instead of a voxel approach the memory demand and pathfinding computing time is reduced. The disadvantage of a distance map is the need for a specific distance map for each distinct goal node.

Hwang and Ahuja (1992) use a potential field to navigate in an octree. The potential field indicates a heuristic potential of each node, in such a field there are local minima (nodes with a low potential). First, a graph is computed between these local minima and a global planner searches a path in the graph. Subsequently, a local planner checks the path for collisions. If a collision is found the path is deleted and the process restarts. A Dijkstra or $\mathrm{A}^{*}$ algorithm is used to navigate the graph.

Broersen et al. (2016) created a simple pathfinding algorithm based on an $A^{*}$ algorithm. The route was computed through the empty space in the octree. The pathfinding method considered two nodes as neighbours if they share a common face. Neighbours could be smaller, larger and of equal size. The neighbours are computed on the fly and object avoidance was not implemented.

Kambhampati and Davis (1985) propose a multi resolution pathfinding method in a quadtree. To reduce the amount of leaf nodes a pruned quadtree is created. In a pruned quadtree, grey nodes containing empty (white) and occupied (black) are possible neighbours.

\subsection{Neighbour finding methods in octree data structures}

All pathfinding algorithms rely on the exploration of adjacent nodes. In an octree structure, two nodes can be each other's neighbour if they share a common: face, edge, or vertex. Figure 3 illustrates the types of connectivity's. In total, there are 26 possible neighbours for each node. This section describes the related work of neighbour finding.
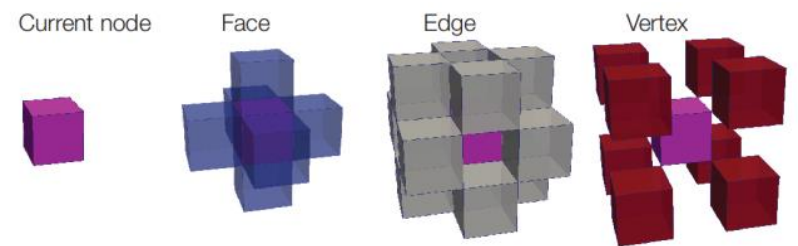

Figure 3: Sharing a common face (6), edge (12), or vertex (8)

In the neighbour finding method of Gargantini (1982) motion is possible in the face direction. Each octant in has a unique location code. This location code describes the location of a node inside the octree. A distinction is made between nodes sharing a common parent and nodes having a different parent node. Adjacent nodes are computed using a separate function for each direction. The main drawback of this method is that the algorithm does not know if a neighbour is part of the octree.

Samet (1989) proposes a method to compute face, edge and vertex neighbours in all possible sizes (smaller, equal, and larger). Neighbours are found by ascending the octree in search of a common ancestor. The neighbouring node is found by descending the tree with mirrored moves.

(Vörös, 2000) improved the method of (Samet, 1989) on three areas. He used the work of Gargantini (1982) to implement the difference between inner and outer neighbours. Using location codes, the octree could be stored as linear area instead of a tree structure. He uses a binary exclusive or operations on the appropriate number of the location code and a directional relevant bit mask to compute neighbours. Xu et al. (2015) checks for face neighbours based on their geometrical location. Payeur (2006) provides a method to search face, edge and vertex neighbours in all possible sizes. For this, a lookup table is used. Namdari et al. (2015) describes a method for neighbour finding during the octree construction. The method is used to find all face, edge and vertex neighbours in all sizes. The method is based on a bread-first search octree generation. Meaning: from the root node to the leaf nodes. The computational effort is minimized by only searching equal and larger neighbours. To make sure also smaller neighbours are stored a neighbouring connection is stored in both directions. If node $\mathrm{X}$ has a neighbour node $\mathrm{Y}$ this neighbour information should be stored in both node $\mathrm{X}$ and $\mathrm{Y}$. This last method ensures that smaller neighbours of a node are found and stored in a later stage in the octree construction. The actual approach in which neighbours are found is quite basic and 
does not take advantage of the locational codes. It just checks if two nodes share $\mathrm{x}, \mathrm{y}$ and/or $\mathrm{z}$ coordinates.

\subsection{Collision avoidance methods}

Next, the related work on collision avoidance is presented: Jung and Gupta (1996) use a distance map for collision detection. For each node the distance to the closest object is calculated. In the method, motion is not limited between centre points of nodes, as instead motion is possible from any point in the octree. This means two distance need to be stored.

Samet (1982) also uses a distance map to create a collision free path in a quadtree. In contrast to Jung and Gupta (1996) motion is only possible between the centre points of the nodes, so only one distance value needs to be stored. An efficient way of finding the closest neighbour is based on the theorem: not all equal sized neighbouring nodes of a white node can be white since merging between the nodes will take place and the white node would not exist.

In a potential field approach, an artificial field is generated in which both the target node and all obstacles direct a force on each empty node in a field. The target node has an attractive force and the obstacles have a repulsive force. These forces are strong at the source and gradually decrease as the distance to the source increase. The sum of these forces are the potential value of a node, together all nodes form a potential field. The maximal potential field is in the obstacles, a low potential field is thus favourable in pathfinding applications (Hou and Zheng, 1994) (Hwang and Ahuja, 1992). Wu and Hori (2006) use a potential field to avoid obstacles. Hamada and Hori (1996) combine a global path planner with al local path planner. Collision detection is performed in the local path planner. Kambhampati and Davis (1985) create a buffer around object to prevent collisions.

\section{METHODOLOGY}

\subsection{Octree generation}

An overview of the method is illustrated in Figure 4. The basic idea of the method is to use an octree to segment a point cloud, where the octree is used as a kind of catalyst for $\mathrm{A}^{*}$ pathfinding. The octree participates the pathfinding process. Before the octree generation the point cloud is geometrical pre-processed. The occupied (non-empty) and empty nodes are generated as described in (Broersen et al., 2016). During the construction of an empty node, it is checked if it is an interior or exterior empty node. For each interior node, the distance to the closest occupied node spatially directly beneath the node is computed. Next, the possible neighbours and connectivity of the interior empty nodes is computed and stored in a network graph. Using the possible neighbours, a collision avoidance system is computed. The network graph and interior empty space are used for $\mathrm{A}^{*}$ pathfinding.

\subsection{Interior empty space}

In this research, only interior point clouds are used for pathfinding. These point clouds are scanned from the inside therefore only the space between a point and the location of the scanner can be classified as empty with certainty (Verbree, 2001). Therefore, it is key to identify the interior empty space for cases like pathfinding and volume calculations.

The goal of identifying interior empty nodes is twofold. Firstly, by identifying the interior empty nodes it is prevented for the exterior empty nodes to be further processed and stored, which reduces the amount of storage and computations. Secondly, only the empty nodes, which are of interest, are used in pathfinding. This avoids the path to exit the interior space via windows or open areas.

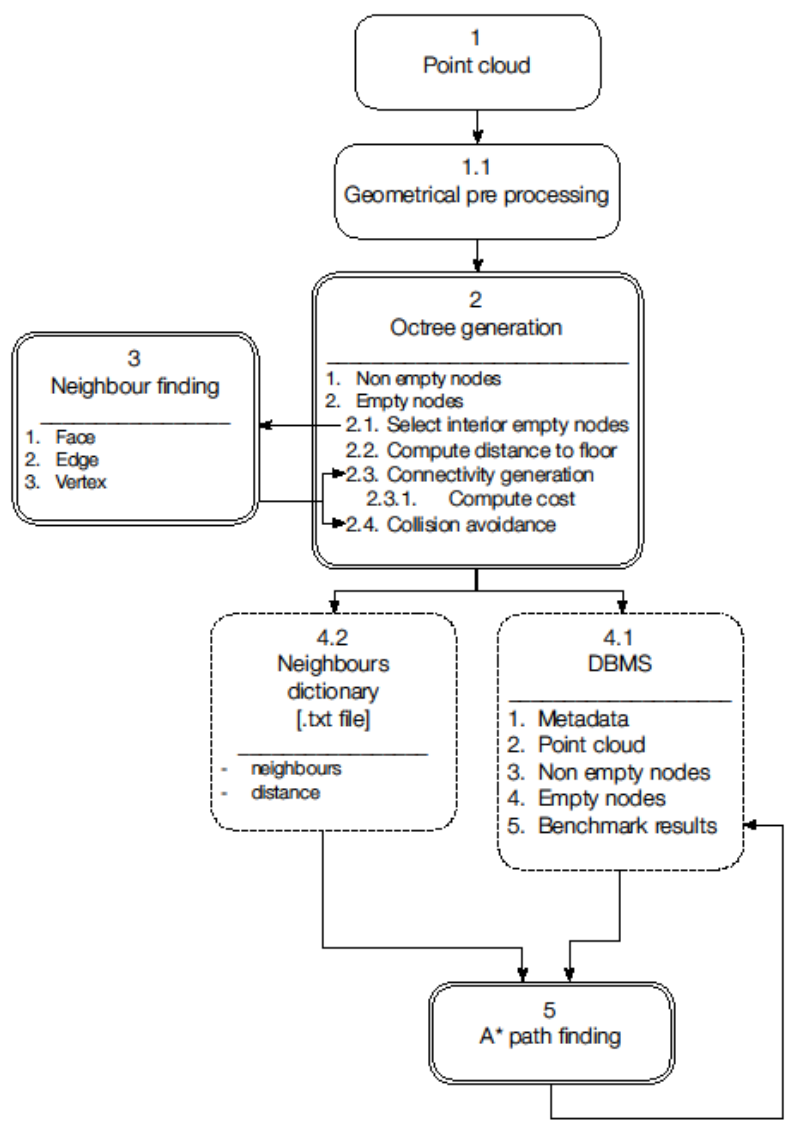

Figure 4: Workflow of the method

An empty node is of the type 'interior empty node' if it has an occupied node spatially straight above it. Figure 5 illustrates a section of a building represented by a quadtree, all nodes that do not have an occupied node (grey) above it are exterior empty nodes (red), and the rest are interior empty nodes (green). To check if a neighbour is interior, neighbours spatially above the node need to be computed until a black node is reached (the node is interior) or until there are no more neighbours (the node is exterior). Only the interior empty nodes are further processed.

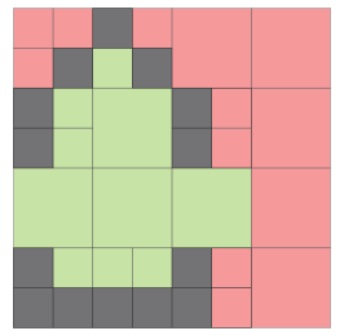

Figure 5: Occupied nodes (grey), interior empty nodes (green), exterior empty nodes (red) 
This method can also be used to compute the distance between an interior empty node and the closest black node directly under it. The distance can be used as a constraint in $\mathrm{A}^{*}$ pathfinding. For example, a maximum distance of 1 meter roughly represent the volume in which a person can reach the closest black node underneath him/her. By selecting a start and goal node maximal 1 meter above a black node the path will likely be bound to the floor. Note that this method is very basic and there is no proof that is will work in all circumstances.

\subsection{Connectivity construction}

To navigate through the empty space of an octree the connectivity between the interior empty nodes must be known. Constructing the connectivity of an interior empty node consist of two steps: first all possible neighbours are computed, next the neighbours that exist as interior empty nodes in the octree are selected.

\subsubsection{Neighbour finding}

The neighbour finding method is based on the work of Vörös (2000), he proposes to find neighbours based on their common ancestor. There are a number of differences between the method of Vörös and the approach used in this research. Firstly, Vörös computes neighbours that are smaller, equal, and larger. In this research only neighbour that are equal and larger are needed, this procedure will be explained in section 3.3.2. Secondly, in the method of Vörös two nodes are neighbours if they share a common face. In this research, two nodes can be neighbours if they share a common: face, edge, and vertex. So the method of Vörös is extended to compute the edge and vertex neighbours. The equal and larger face neighbours are computed like the method of Vörös. The method to compute larger neighbours work the same for face, edge, and vertex neighbours.

The basic idea for finding edge neighbours is: the edge neighbours of node $c$ have a face connection with a face neighbour of node $c$. The method is divided into four steps, Figure 6 illustrates these steps. In the first step, the face neighbours of node $c$ are computed. In the second step the face neighbour which are computed in the directions $x$ and $y$ are selected. In the third step the neighbours in $z$ and $x$ are computed of the face neighbours in direction $x$. In addition, the neighbours in $z$ of the face neighbours in direction $y$ are computed. The computed neighbours of the face neighbours form the complete set of equal edge neighbours.
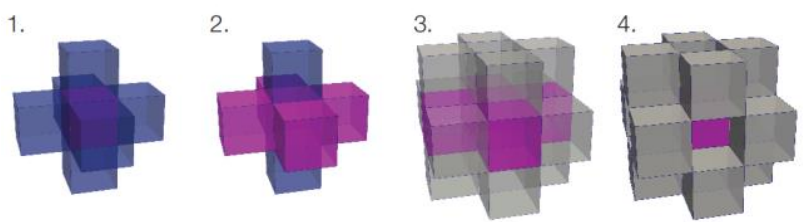

Figure 6: Edge neighbours - first step: face neighbours, second step: selected face neighbours, third step: compute face

The method for computing equal vertex neighbours is similar to that of edge neighbours. Vertex neighbours of node $c$ have a face connection with an edge neighbour of node $c$. The method is divided into four steps, Figure 7 illustrates the steps. In the first step, the edge neighbours are computed. Next the edge neighbours which were computed in direction $x$ are selected. Of the selected node, the face neighbours in the direction $z$ are computed: these nodes are the vertex neighbours.
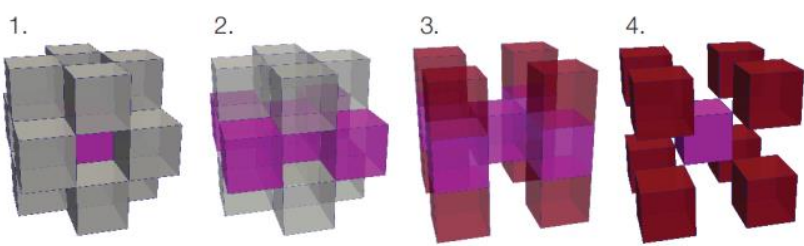

Figure 7: Face neighbours - First step: edge neighbours, second step: selected edge neighbours, third step: compute face

\subsubsection{Connectivity generation}

In this step, the neighbours computed in the section 3.3.1 are used to select neighbours that are interior empty nodes. All occupied nodes are filtered out. The method of Namdari et al. (2015) is used to compute neighbours during bread-first search octree generation. In the method, only equal and larger neighbours are computed. By storing the connection of a larger neighbour in both connected nodes, also smaller neighbours are found in a later stage of the octree generation. Like the method of Namdari et al. (2015), the octree used in this research is computed in a top down approach. For each white node that is computed, the equal and larger neighbours are computed based on the method described in in the previous part of this section. For each larger neighbour the connection is stored in both directions if the larger neighbour is an empty node. For example, if node $\mathrm{x}$ has a larger neighbours $\mathrm{y}$ the connection in node $\mathrm{x}$ and node $\mathrm{y}$ is stored. This step ensures that for the larger node $y$ the smaller neighbours are stored. For each connection, the cost is computed and stored in a network graph.

\subsection{Collision avoidance}

The goal of collision avoidance is to compute a path for an object in which a collision is not possible. A path is collision free if the object does not intersect with any occupied node along the path. For this, we need to know: can an object, with a certain size, fit in an interior empty node, and can this object move between two neighbouring empty nodes. Therefore this section consist of two parts: the first part explains how to compute the distance from the centre of an interior empty node to the closest border with an occupied node. In addition, the second part explains how to compute the distance between a crossing point and the closest black node.

The method of Samet (1982) is used to compute the clearance for each empty node. He describes a theorem that states: All neighbours of a white node cannot all be white because merging would take place and the node would not exist.

The closest boundary with an occupied node is computed with a chessboard distance. Thus the closest occupied node must be, or a descendant of, one of the equal neighbours (see Figure 8). Since occupied nodes are always stored in the lowest level, an occupied node can never be a larger neighbour. So the 26 equal neighbours are needed to find the closest boundary with an occupied node. Since these neighbours were computed and stored during the connectivity generation these are already available. To acquire the closest boundary the set of neighbours is compared with the set of occupied nodes. For each occupied node intersecting with the neighbours, the distance between the border of the occupied node and the centre point of the empty node is computed. As it is possible for multiple occupied nodes to intersect, it is the smallest distance that defines the clearance. The clearance of all interior empty nodes form a clearance map. 


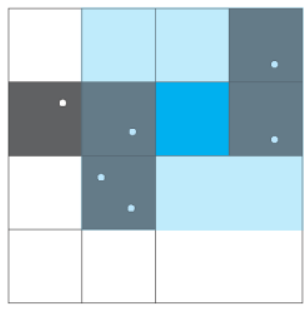

Figure 8: The closest border with a black node and the dark blue node must lie in the light blue area

Figure 9 shows two diagrams, in the left, an object (blue circle) is in the centre of an empty node where the clearance is sufficient. Although the clearance is sufficient in the centre point of a node, the object collides with an occupied node (red) when moving between two empty nodes. For this reason it is key to check the maximal crossing value for each connection between two empty nodes. This crossing value can never be bigger than the minimal clearance of the two connection empty nodes. Otherwise, an object would be able to enter a node with a clearance smaller than the object size and a collision would occur.
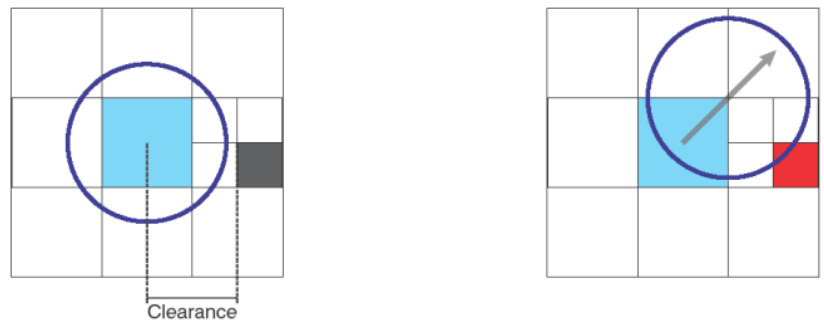

Figure 9: Collision avoidance: Centre point is collision free (left), but movement collides (right)

The next step is to compute the maximal crossing value between two neighbouring interior empty nodes. Figure 10 shows an object on an intersection between two adjacent empty nodes. The only place in the intersection where the distance to a black node can be smaller than the clearance of one of the two empty nodes is in the two red nodes. To find the closest occupied node the nodes in the red areas are compared with the set of occupied nodes. For each occupied node that intersects with the red area the distance to the crossing is computed. In general, the minimal distance is the crossing value. However, if the minimal distance exceeds the clearance of one of the nodes the minimal clearance defines the maximal crossing value. Also, if no occupied nodes are found in the area the maximal crossing value is defined by the minimal clearance. The crossing value is stored in a network graph aside the cost between two empty nodes.
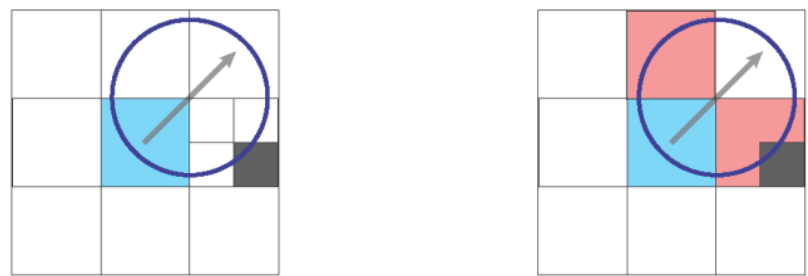

Figure 10: Collision avoidance: The area that must be checked for occupied nodes

\section{TEST AND RESULTS}

\subsection{Point cloud processing}

The point cloud is geometrical pre-processed in the following order: The point cloud is first rotated so it is aligned with the axis. Next, the rotated point cloud is translated so the origin of the point cloud has coordinate $(0,0,0)$. Finally, the point cloud is scaled so it fits best in an octree grid of $2 n * 2 n * 2 n$. Where $n$ refers to the octree depth. Figure 11 illustrates this process.

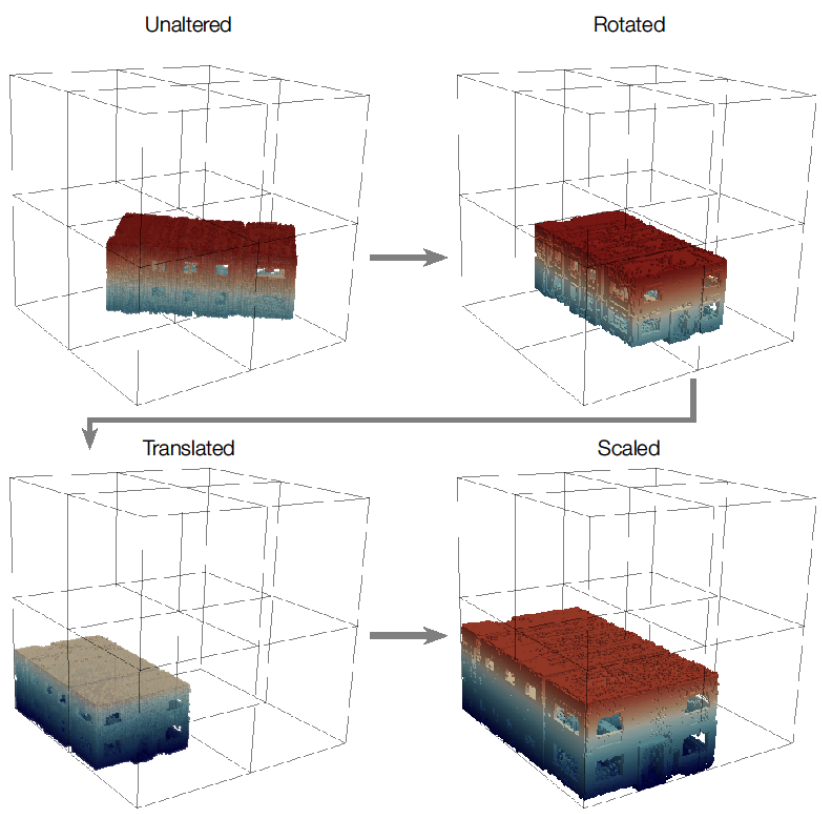

Figure 11: Pre-processing the point cloud

\subsection{Filtering interior empty nodes}

During the octree generation the interior and exterior empty space are identified. All exterior empty space is no further processed. Figure 12 illustrates the difference between the interior and exterior empty space. In this example, 5699 exterior empty space nodes are filtered out on 11348 interior empty nodes.

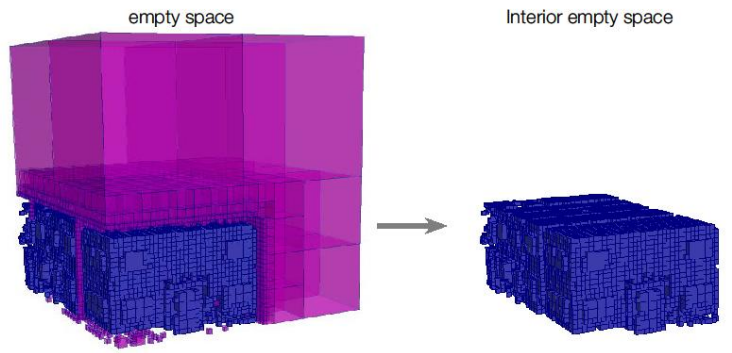

Figure 12: Filtering of interior empty nodes

\subsection{Octree generation}

Figure 1 illustrates the total process of identifying the interior empty nodes of a point cloud. The octree in Figure 1 is created from a point cloud of 2.196 .903 points with 6 octree levels.

The computer used in the test has an Intel(R) Core(TM) i5-4590 CPU @3.30GHz processor, 8.00 GB RAM running Windows 10 Pro 64 bits. 
All scripts are written in the programming language 'python'. For the database management, postgreSQL was used. To connect Python to postgreSQL, the package psycopg2 is used. The package LibLas is used to work with .las files (point clouds) in python. For visualizations, the open source software Paraview is used.

\subsection{Connectivity generation}

Figure 13 illustrates the process of generating the connectivity of a current interior empty node. First all possible neighbours are computed. Finally, all nodes in the possible neighbours intersecting with an occupied node are excluded. Besides, a neighbour is always stored as big as possible; this excludes neighbours that are children nodes of bigger neighbours.

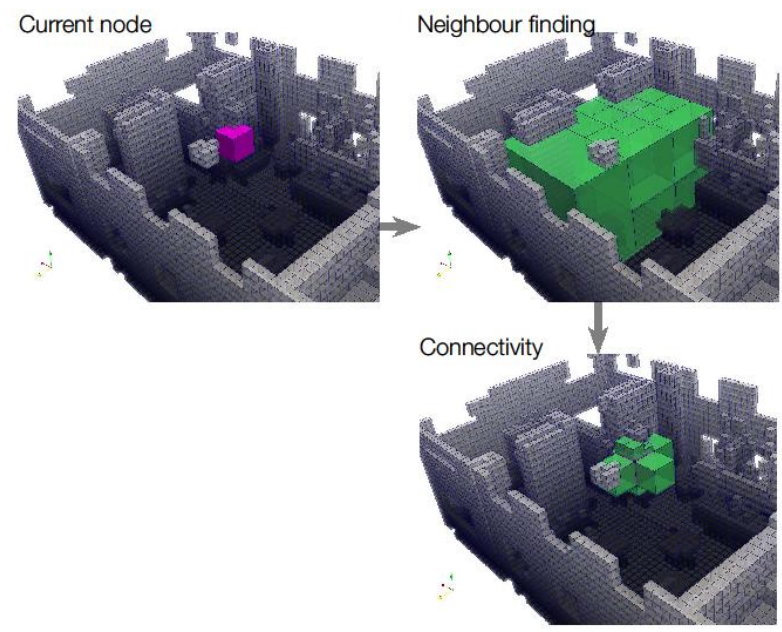

Figure 13: Connectivity generation

\subsection{Pathfinding}

The computed interior empty nodes and network graph can now be used for A* pathfinding. Figure 14 illustrates two path through the interior empty space of a point cloud.

All empty nodes closer than $1 \mathrm{~m}$ to the closest black node directly under it are selected for pathfinding. Further, only empty nodes that have a clearance higher than $0.17 \mathrm{~m}$ are selected, this ensure that in each node an object of $0.34 \mathrm{~m}$ is collision free. Finally, movement between two nodes is only possible if the crossing value is higher than $0.17 \mathrm{~m}$, this way also the crossing points are collision free. A path is computed on average in about 0.8 second.

\section{DISCUSSION}

This paper presents a new workflow for pathfinding in a point cloud. The point cloud is processed so it fits best in an octree. During the octree generation, the interior empty nodes are selected and further processed. For each interior empty node the distance to the closest occupied node, spatially directly under it is computed. Next, a network graph is computed for all empty nodes. The connectivity for each node consist of all possible neighbours (face, edge and vertex and in all sizes). Besides, a collision avoidance system is pre-processed. This system consist of two parts. First, the clearance of each empty node is computed, and next the maximal crossing value between two empty neighbouring nodes is computed. Finally, the empty nodes and network graph are used in $\mathrm{A}^{*}$ pathfinding.
Translating and rotating a point cloud aligns it for scaling. Scaling the point cloud create the best circumstances for segmentation is an octree structure. Using the clearance and crossing value collision free pathfinding in a point cloud is possible. If a path should be bound to the floor the distance to the closest black node under a node can be used as constraint in $\mathrm{A}^{*}$ pathfinding.

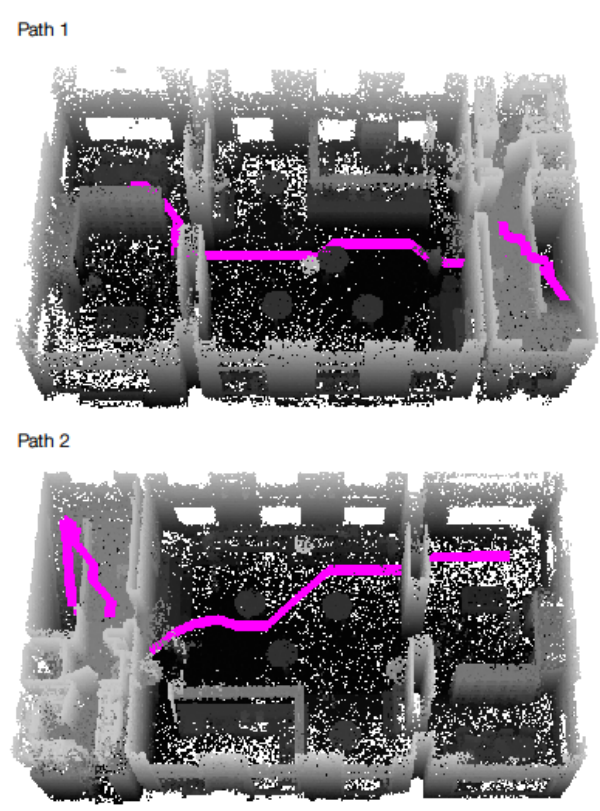

Figure 14: Pathfinding in the empty space of a point cloud

\subsection{Future work}

This point clouds in this research were of small buildings $(20 \mathrm{~m} * 20 \mathrm{~m} * 10 \mathrm{~m})$. Both pathfinding and octree generation is relatively fast. The method can be more beneficial in larger buildings. More research should be conducted to check the possibilities of the method on large building (conference halls, station).

If a Dijkstra algorithm is used instead of an $\mathrm{A}^{*}$ algorithm it is possible to find all possible locations from a current node. In emergency, this can be useful to find the closest exit. For this, all nodes close to an exit should be semantically enriched.

In this research, the point cloud was rotated to align with the axis manually. To make the method completely automatic more research should be conducted. One possible approach could be to use RANSAC plane fitting to identify large vertical planes and rotate the point cloud to follow the plane.

\section{REFERENCES}

Broersen, T., Fichtner, F. W., Heeres, E. J., de Liefde, I., Rodenberg, O. B. P. M., Verbree, E., and Voûte, R. (2016). Project pointless. Identifying, visualising and pathfinding through empty space in interior point clouds using an octree approach. In AGILE 2016; 19th AGILE Conference on Geographic Information Science, 14-17 June, 2016; Authors version. 
Gargantini, I. (1982). Linear octrees for fast processing of threedimensional objects. Computer graphics and Image processing, 20(4):365-374. Guang-lei, Z. and He-Ming, J. (2012). Global path planning of AUV based on improved ant colony optimization algorithm. In Automation and Logistics (ICAL), 2012 IEEE International Conference on, pages 606-610. IEEE.

Hamada, K. and Hori, Y. (1996). Octree-based approach to realtime collision-free path planning for robot manipulator. In Advanced Motion Control, 1996. AMC'96-MIE. Proceedings 1996 4th International Workshop, volume 2, pages 705-710. IEEE.

Hart, P. E., Nilsson, N. J., and Raphael, B. (1968). A formal basis for the heuristic determination of minimum cost paths. Systems Science and Cybernetics, IEEE Transactions on, 4(2):100-107.

Herman, M. (1986). Fast, three-dimensional, collision-free motion planning. In Robotics and Automation. Proceedings. 1986 IEEE International Conference on, volume 3, pages 10561063. IEEE.

Hou, E. S. and Zheng, D. (1994). Mobile robot path planning based on hierarchical hexagonal decomposition and artificial potential fields. Journal of Robotic Systems, 11(7):605-614.

Hwang, Y. K. and Ahuja, N. (1992). A potential field approach to path planning. Robotics and Automation, IEEE Transactions on, 8(1):23-32.

Isikdag, U., Zlatanova, S., and Underwood, J. (2013). A BIMOriented Model for supporting indoor navigation requirements. Computers, Environment and Urban Systems, 41:112-123.

Jung, D. and Gupta, K. K. (1996). Octree-based hierarchical distance maps for collision detection. In Robotics and Automation, 1996. Proceedings., 1996 IEEE International Conference on, volume 1, pages 454-459. IEEE.

Kambhampati, S. and Davis, L. S. (1985). Multiresolution path planning for mobile robots. Technical report, DTIC Document.

Kim, J. and Lee, S. (2009). Fast neighbor cells finding method for multiple octree representation. In 2009 IEEE International Symposium on Computational Intelligence in Robotics and Automation - (CIRA).

Namdari, M. H., Hejazi, S. R., and Palhang, M. (2015). Mcpn, octree neighbor finding during tree model construction using parental neighboring rule. 3D Research, 6(3):1-15.

Payeur, P. (2006). A computational technique for free space localization in 3- $\mathrm{d}$ multiresolution probabilistic environment models. Instrumentation and Measurement, IEEE Transactions on, 55(5):1734-1746.

Samet, H. (1982). Distance transform for images represented by quadtrees. Pattern Analysis and Machine Intelligence, IEEE Transactions on, (3):298-303.

Samet, H. (1989). Neighbor finding in images represented by octrees. Computer Vision, Graphics, and Image Processing, 46(3):367-386. van Oosterom, P. and Vijlbrief, T. (1996). The spatial location code. In Proceedings of the 7 th international symposium on spatial data handling, Delft, The Netherlands.
Verbree, E. (2003). The STIN Method: 3D-Surface reconstruction by observation lines and Delaunay TENs, ISPRS Archives - Volume XXXIV-3/W13, 2003

Vörös, J. (2000). A strategy for repetitive neighbor finding in octree representations. Image and Vision Computing, 18(14):1085-1091.

Vörös, J. (2001). Low-cost implementation of distance maps for path planning using matrix quadtrees and octrees. Robotics and Computer-Integrated Manufacturing, 17(6):447-459.

Wu, L. and Hori, Y. (2006). Real-time collision-free path planning for robot manipulator based on octree model. In Advanced Motion Control, 2006. 9th IEEE International Workshop on, pages 284-288. IEEE.

Xu, S., Honegger, D., Pollefeys, M., and Heng, L. (2015). Realtime $3 \mathrm{~d}$ navigation for autonomous vision-guided mavs. IROS. 